\title{
Implementation and Use of a Convergence Test for Iterative Wind Tunnel Balance Load Predictions
}

\author{
N. Ulbrich ${ }^{\dagger}$ \\ Jacobs Technology Inc., Moffett Field, California 94035
}

\begin{abstract}
A modified and extended version of a convergence test for wind tunnel strain-gage balance load iterations was implemented. The test uses an upper bound of the Lipschitz constant to assess convergence characteristics of balance load predictions if the Iterative Method is applied. Convergence is expected within the use envelope of the balance whenever this upper bound is less than the threshold of one. It is explained in great detail how the convergence test can be applied to the two load iteration equation types that are currently being used in the aerospace testing community. In addition, the application of the test to balances with bi-directional output characteristics is discussed. It is also shown how numerical differentiation can be used to obtain partial derivatives that are needed for the calculation of the Lipschitz constant. Finally, machine calibration data of NASA's MC60E six-component force balance is selected to demonstrate both implementation and use of the convergence test.
\end{abstract}

\section{Nomenclature}

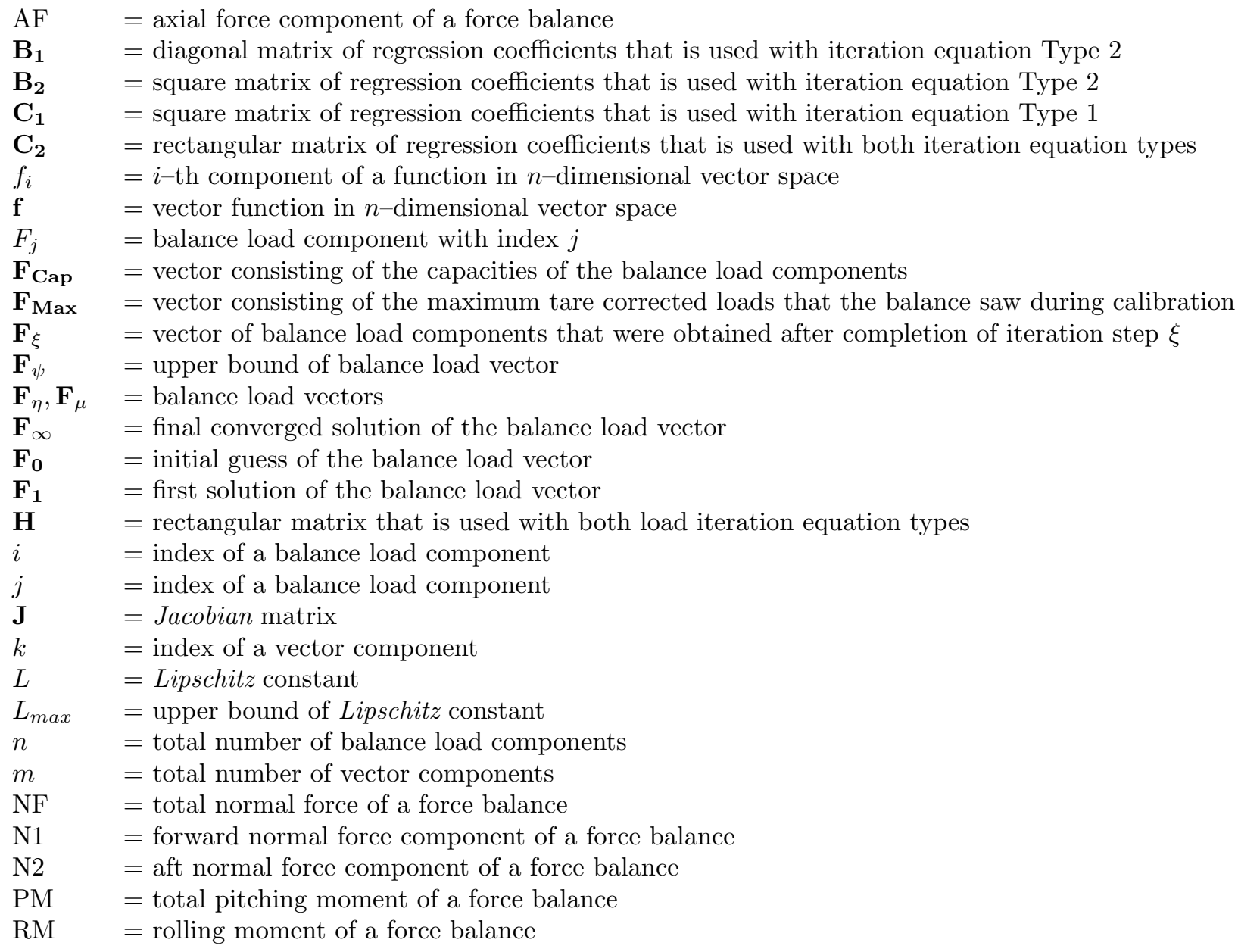

\footnotetext{
$\dagger$ Aerodynamicist, Jacobs Technology Inc.
} 


$\begin{array}{ll}\mathrm{SF} & =\text { total side force of a force balance } \\ \mathrm{S} 1 & =\text { forward side force component of a force balance } \\ \mathrm{S} 2 & =\text { aft side force component of a force balance } \\ \mathbf{X} & =\text { vector } \\ \mathrm{YM} & =\text { total yawing moment of a force balance } \\ \Delta F_{j} & =\text { optimum step size for the application of the central difference formula } \\ \Delta \mathbf{R} & =\text { vector of gage output differences of an } n-\text { component balance } \\ \varepsilon & =\text { relative machine precision at runtime } \\ \Theta & =\text { load iteration tolerance expressed as a percentage of a fixed reference load } \\ \lambda & =\text { characteristic scale } \\ \xi & =\text { iteration step index }\end{array}$

\section{Introduction}

In general, a load iteration is required to predict wind tunnel strain-gage balance loads from the measured gage outputs during a wind tunnel test if the Iterative Method is used to analyzed and process balance calibration data. The need for the use of a load iteration scheme results from the fact that the Iterative Method fits the gage outputs as a function of the balance loads during the regression analysis of balance calibration data (see, e.g., Refs. [1], [2], and [3] for more details about the Iterative Method).

Two types of load iteration equations, i.e., Type 1 and Type 2, can be developed from the regression models of the gage outputs to predict the balance loads. Iteration Equation Type 1 is more universally applicable. It will converge if the balance loads are expressed in either the "design" format of the balance (e.g., loads of a force balance are described as N1, N2, S1, S2, RM, AF) or in "direct-read" format (e.g., loads of a force balance are described as NF, PM, SF, YM, RM, AF) as long as the regression models of the gage outputs do not have hidden linear dependencies. Type 2, on the other hand, is more limited. In that case, loads must always be expressed in the "design" format of the balance to guarantee convergence (see Ref. [4] for a detailed discussion of this limitation). In general, load predictions obtained from the two iteration equation types are identical for all practical purposes as long as the iteration equations were obtained from the same regression models of the outputs. Table 1 below lists the two iteration equation types in matrix format where $\mathbf{F}$ is the load vector, $\boldsymbol{\Delta} \mathbf{R}$ is the vector of output differences, $\xi$ is the iteration step index, and $\mathbf{B}_{\mathbf{1}}, \mathbf{B}_{\mathbf{2}}, \mathbf{C}_{\mathbf{1}}$, and $\mathbf{C}_{\mathbf{2}}$ are the coefficient matrices (see Refs. [1], [2], and [3] for more details; highly detailed derivations of the two load iteration equations can be found in Refs. [2] and [3]).

Table 1: Iteration Equation Types for Strain-Gage Balance Load Prediction.

\begin{tabular}{|c|c|c|}
\hline TYPE & ITERATION EQUATION DEFINITION & $\begin{array}{l}\mathbf{F}_{\mathbf{0}} \equiv \text { INITIAL GUESS } \\
\mathbf{F}_{1} \equiv \text { FIRST SOLUTION }\end{array}$ \\
\hline 1 & $\begin{array}{c}\mathbf{F}_{\xi}=\mathbf{C}_{\mathbf{1}}^{-1} \mathbf{\Delta} \mathbf{R}-\left\{\mathbf{C}_{\mathbf{1}}^{-1} \mathbf{C}_{\mathbf{2}} \cdot \mathbf{H}\left(\mathbf{F}_{\xi-1}\right)\right\} \\
\ldots \text { taken from Ref. [1], Eq. (3.3.7), or, Ref. [2], Eq. (30) }\end{array}$ & $\begin{array}{l}\mathbf{F}_{\mathbf{0}}=\left[\begin{array}{c}0 \\
\vdots \\
0\end{array}\right] \\
\mathbf{F}_{\mathbf{1}}=\mathbf{C}_{\mathbf{1}}^{-1} \Delta \mathbf{R}\end{array}$ \\
\hline 2 & $\begin{array}{c}\mathbf{F}_{\xi}=\mathbf{B}_{\mathbf{1}}^{-1} \boldsymbol{\Delta} \mathbf{R}-\left\{\mathbf{B}_{\mathbf{1}}^{-1} \mathbf{B}_{\mathbf{2}} \cdot \mathbf{F}_{\xi-\mathbf{1}}+\mathbf{B}_{\mathbf{1}}^{-1} \mathbf{C}_{\mathbf{2}} \cdot \mathbf{H}\left(\mathbf{F}_{\xi-\mathbf{1}}\right)\right\} \\
\ldots \text { taken from Ref. [3], Eq. }(26)\end{array}$ & $\begin{array}{l}\mathbf{F}_{\mathbf{0}}=\left[\begin{array}{c}0 \\
\vdots \\
0\end{array}\right] \\
\mathbf{F}_{\mathbf{1}}=\mathbf{B}_{\mathbf{1}}^{-1} \boldsymbol{\Delta} \mathbf{R}\end{array}$ \\
\hline
\end{tabular}


The coefficients of the two iteration equation types above are typically stored in a so-called "data reduction matrix." It was already shown by Smith in the early 1970s that these coefficients may be used in combination with a reasonable estimate of the upper bound of the load vector of the balance to evaluate the expected convergence characteristics of the iteration equation within the expected use envelope of the balance (for more detail see Ref. [5]). However, Smith only applied his convergence test to iteration equation Type 1. He also did not discuss the application of his approach to balances with bi-directional gage outputs. Furthermore, no examples were provided that show load iteration convergence test results for typical balances. Finally, Smith used explicit solutions of the partial derivatives of the iteration equations as input for his convergence test. This traditional approach to differentiation is more complex to implement and maintain in a balance data analysis software package. Therefore, the author decided to extend and modify Smith's original convergence test in order to address some of its limitations. There modifications are described in great detail in the current paper.

First, modifications and extensions of Smith's original convergence test are presented. Afterwards, data from the machine calibration of a six-component force balance is used to illustrate the application of the convergence test for six different balance data analysis situations.

\section{Modification and Implementation of Convergence Test}

\section{A. Iteration Convergence Test}

It is shown in this section how Smith's original iteration convergence test can be modified so that the test works with both iteration equation types that are used in the aerospace testing community. First, the convergence test itself is reviewed. Smith correctly suggested to use an estimate of the upper bound of the Lipschitz constant for the evaluation of the convergence characteristics of the load iterations (see Ref. [5] for more detail). In general, Smith's derivations closely follow steps that are described in Henrici's textbook on numerical analysis. Henrici investigated the convergence characteristics of the following vector "sequence" (see Ref. [6], p. 99, Eq. (95-6)) ...

$$
\mathbf{F}_{\xi}=\mathbf{f}\left(\mathbf{F}_{\xi-1}\right)
$$

where

$$
\mathbf{F}_{\xi}=\left[\begin{array}{c}
F_{1} \\
F_{2} \\
\vdots \\
F_{n}
\end{array}\right]
$$

and $\xi$ is assumed to be the "sequence" (iteration) step index. Vector $\mathbf{F}_{\xi}$ can be interpreted as the load vector of an $n$-component balance. Then, $F_{1}$ to $F_{n}$ are the load components of the balance. In addition, the right-hand side of Eq. (1a) may be interpreted as the right-hand side of a load iteration equation that is listed in Table 1 above. Consequently, we can describe the right-hand side of Eq. (1a) as follows:

$$
\mathbf{f}\left(\mathbf{F}_{\xi-\mathbf{1}}\right)=\left[\begin{array}{c}
f_{1}\left(\mathbf{F}_{\xi-\mathbf{1}}\right) \\
f_{2}\left(\mathbf{F}_{\xi-\mathbf{1}}\right) \\
\vdots \\
f_{n}\left(\mathbf{F}_{\xi-\mathbf{1}}\right)
\end{array}\right]=\left\{\begin{array}{l}
\underbrace{\mathbf{C}_{\mathbf{1}}^{-1} \Delta \mathbf{R}-\left\{\mathbf{C}_{\mathbf{1}}^{-1} \mathbf{C}_{\mathbf{2}} \cdot \mathbf{H}\left(\mathbf{F}_{\xi-1}\right)\right\}}_{\text {Iteration Equation Type } 1} \\
\underbrace{\mathbf{B}_{\mathbf{1}}^{-1} \Delta \mathbf{R}-\left\{\mathbf{B}_{\mathbf{1}}^{-1} \mathbf{B}_{\mathbf{2}} \cdot \mathbf{F}_{\xi-\mathbf{1}}+\mathbf{B}_{\mathbf{1}}^{-1} \mathbf{C}_{\mathbf{2}} \cdot \mathbf{H}\left(\mathbf{F}_{\xi-\mathbf{1}}\right)\right\}}_{\text {Iteration Equation Type 2 }}
\end{array}\right.
$$

Henrici rigorously showed in his textbook that the sequence defined in Eq. (1a) above will converge to a single value if the Lipschitz constant of the sequence, i.e., of the load iteration process, is less than the threshold of one (see Ref. [6], p. 99-101). This convergence test can be summarized as follows: 


\section{CONDITION FOR CONVERGENCE OF THE LOAD ITERATION PROCESS}

$$
\begin{gathered}
0 \leq L<1 \\
\text { where } \\
L \equiv \text { Lipschitz constant }
\end{gathered}
$$

In theory, Henrici's convergence test can directly be applied to the load iteration process if a "conservative" upper bound of the Lipschitz constant is obtained from (i) a suitable upper bound of the load vector and (ii) upper bounds of the partial derivatives of the load iteration equations with respect to the individual load components of the balance. Then, convergence of the load iterations is guaranteed within the use envelope of the balance as long as the "conservative" upper bound of the Lipschitz constant is less than one. This conclusion is summarized in Eq. (4) below where the upper bound of the partial derivatives

CONSERVATIVE UPPER BOUND OF THE LIPSCHITZ CONSTANT

$$
\begin{gathered}
L \leq L_{\max }=\sqrt{\sum_{i=1}^{n} \sum_{j=1}^{n}\left[\left\{\frac{\partial f_{i}\left(\mathbf{F}_{\psi}\right)}{\partial F_{j}}\right\}_{\max }\right]^{2}}<1 \\
\text { where } \\
\mathbf{F}_{\psi} \equiv \text { upper bound of the load vector } \\
\left\{\frac{\partial f_{i}\left(\mathbf{F}_{\psi}\right)}{\partial F_{j}}\right\}_{\max } \equiv \text { upper bound of coefficient of the Jacobian matrix } \mathbf{J}\left\{\mathbf{f}\left(\mathbf{F}_{\psi}\right)\right\}
\end{gathered}
$$

is obtained from the Jacobian matrix of the right-hand sides of the load iteration equations that are described in Table 1. Three different choices are suggested in the literature in order to define an upper bound of the load vector (see Ref. [5], App. B). A suitable choice is either a function of the capacities $\left(\mathbf{F}_{\mathbf{C a p}}\right)$ of the balance load components, or, a function of the maximum tare corrected loads $\left(\mathbf{F}_{\mathbf{M a x}}\right)$ that the balance experienced during its calibration. These literature recommended options can be described as follows:

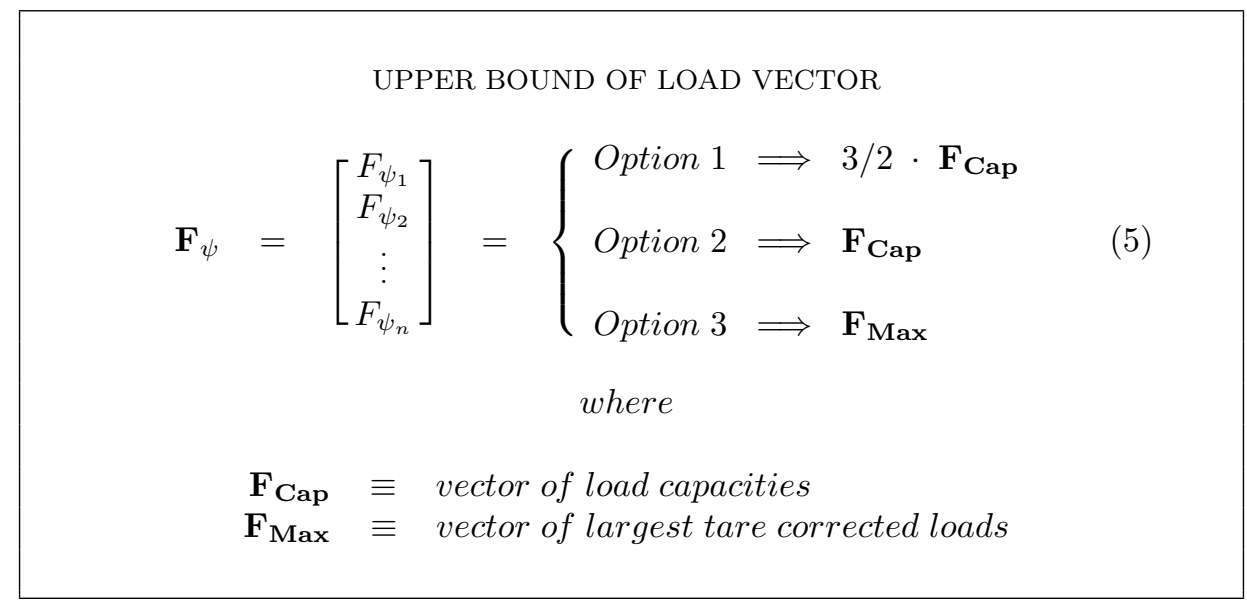


It is important to point out that the suggested upper bounds of the load vector are very "conservative" in nature. They imply that the iteration is performed while all load components are simultaneously loaded near their design maximum. This situation practically never occurs during the actual use of the balance.

The determination of upper bounds of the partial derivatives of the iteration equation is more complex. In principle, the Jacobian matrix of a load iteration equation is an $n \times n$ matrix for an $n$-component balance. Therefore, assuming, for example, that the upper bound of the Lipschitz constant is to be estimated for a six-component balance, a total of 36 partial derivatives have to be computed. For improved clarity, the Jacobian matrix may be described as a collection of $n$ column vectors. Then, we get

$$
\mathbf{J}\left\{\mathbf{f}\left(\mathbf{F}_{\psi}\right)\right\}=\left[\begin{array}{llllll}
\frac{\partial \mathbf{f}\left(\mathbf{F}_{\psi}\right)}{\partial F_{1}} & \frac{\partial \mathbf{f}\left(\mathbf{F}_{\psi}\right)}{\partial F_{2}} & \ldots & \frac{\partial \mathbf{f}\left(\mathbf{F}_{\psi}\right)}{\partial F_{j}} & \ldots & \frac{\partial \mathbf{f}\left(\mathbf{F}_{\psi}\right)}{\partial F_{n}}
\end{array}\right]
$$

where a column vector associated with the derivative $\partial / \partial F_{j}$ is defined as the corresponding vector of derivatives of the right-hand side of the iteration equation that is given in Eq. (2). Then, knowing that $\partial / \partial F_{j}$ of the vectors $\mathbf{C}_{\mathbf{1}}^{-1} \Delta \mathbf{R}$ and $\mathbf{B}_{\mathbf{1}}^{-1} \Delta \mathbf{R}$ equals zero because both vectors are independent of $F_{j}$, we get:

$$
\frac{\partial \mathbf{f}\left(\mathbf{F}_{\psi}\right)}{\partial F_{j}}=\left[\begin{array}{c}
\frac{\partial f_{1}\left(\mathbf{F}_{\psi}\right)}{\partial F_{j}} \\
\vdots \\
\frac{\partial f_{i}\left(\mathbf{F}_{\psi}\right)}{\partial F_{j}} \\
\vdots \\
\frac{\partial f_{n}\left(\mathbf{F}_{\psi}\right)}{\partial F_{j}}
\end{array}\right]=\left\{\begin{array}{c}
\underbrace{-\frac{\partial}{\partial F_{j}}\left\{\mathbf{C}_{\mathbf{1}}^{-1} \mathbf{C}_{\mathbf{2}} \cdot \mathbf{H}\left(\mathbf{F}_{\psi}\right)\right\}}_{\text {Iteration Equation Type } 2} \\
\underbrace{-\frac{\partial}{\partial F_{j}}\left\{\mathbf{B}_{1}^{-1} \mathbf{B}_{\mathbf{2}} \cdot \mathbf{F}_{\psi}+\mathbf{B}_{\mathbf{1}}^{-1} \mathbf{C}_{2} \cdot \mathbf{H}\left(\mathbf{F}_{\psi}\right)\right\}}_{\text {Iteration Equation Type } 1}
\end{array}\right.
$$

Now, an upper bound of the partial derivatives shown in Eq. (6b) above can be obtained by simply using the absolute values of all constants and derivatives on the right-hand side of Eq. (6b). This conclusion can be expressed as follows...

$$
\frac{\partial \mathbf{f}\left(\mathbf{F}_{\psi}\right)}{\partial F_{j}} \leq\left\{\frac{\partial \mathbf{f}\left(\mathbf{F}_{\psi}\right)}{\partial F_{j}}\right\}_{\text {max }}=\left\{\begin{array}{l}
\underbrace{\frac{\partial}{\partial F_{j}}\left\{\left|\mathbf{C}_{\mathbf{1}}^{-1} \mathbf{C}_{\mathbf{2}}\right| \cdot\left|\mathbf{H}\left(\mathbf{F}_{\psi}\right)\right|\right\}}_{\text {Iteration Equation Type 1 }} \\
\underbrace{\frac{\partial}{\partial F_{j}}\left\{\left|\mathbf{B}_{\mathbf{1}}^{-1} \mathbf{B}_{\mathbf{2}}\right| \cdot\left|\mathbf{F}_{\psi}\right|+\left|\mathbf{B}_{\mathbf{1}}^{-1} \mathbf{C}_{\mathbf{2}}\right| \cdot\left|\mathbf{H}\left(\mathbf{F}_{\psi}\right)\right|\right\}}_{\text {Iteration Equation Type 2 }}
\end{array}\right.
$$

where the vector or matrix operator symbol $|\ldots|$ simply indicates that the absolute value of the vector or matrix coefficient is used. The vector of partial derivatives listed in Eq. (7) still needs to be computed by using the iteration equations listed in Table 1 as input. Smith explicitly calculated those derivatives (see Ref. [5], program listing on p. 23). His approach, however, may be complex to implement in a data analysis program that supports (i) both iteration equation types and (ii) all ten math term group choices that are recommended in the literature for the regression model of a gage output (see Ref. [1], p. 9, Eq. (3.1.3)). Instead, it may be easier to simply compute the derivatives numerically by using the central difference scheme. This alternate approach is described in the next section of the paper.

It must also be mentioned for completeness that the regression model of a gage output and, consequently, the load iteration equation itself, can have absolute value terms of the loads if "bi-directional' output characteristics of a balance are modeled (see Ref. [1], pp. 8-9, Eq. (3.1.3)). In that case, a subset of the partial derivatives is not defined at or near zero absolute load. Fortunately, the partial derivatives used in Eq. (7) are evaluated for the upper bound $\mathbf{F}_{\psi}$ of the load vector. Its components are large positive values that 
are in the neighborhood of the load capacities. Therefore, the suggested numerical calculation of the partial derivatives will work and, as a result, the influence of the absolute value terms on the overall convergence characteristics of the load iteration equation is included in the convergence test.

\section{B. Numerical Calculation of Partial Derivatives}

It is possible to compute the partial derivatives $\left\{\partial f_{i}\left(\mathbf{F}_{\psi}\right) / \partial F_{j}\right\}_{\max }$ numerically by using the central difference scheme. Therefore, in order to better describe the application of the central difference scheme to Eq. (7), an auxiliary vector $\mathbf{g}\left(\mathbf{F}_{\psi}\right)$ is introduced that is defined as follows ...

$$
\mathbf{g}\left(\mathbf{F}_{\psi}\right)=\left[\begin{array}{c}
g_{1}\left(\mathbf{F}_{\psi}\right) \\
\vdots \\
g_{i}\left(\mathbf{F}_{\psi}\right) \\
\vdots \\
g_{n}\left(\mathbf{F}_{\psi}\right)
\end{array}\right]=\left\{\begin{array}{l}
\underbrace{\left|\mathbf{C}_{\mathbf{1}}^{-1} \mathbf{C}_{\mathbf{2}}\right| \cdot\left|\mathbf{H}\left(\mathbf{F}_{\psi}\right)\right|}_{\text {Iteration Equation Type 1 }} \\
\underbrace{\left|\mathbf{B}_{\mathbf{1}}^{-1} \mathbf{B}_{\mathbf{2}}\right| \cdot\left|\mathbf{F}_{\psi}\right|+\left|\mathbf{B}_{\mathbf{1}}^{-1} \mathbf{C}_{\mathbf{2}}\right| \cdot\left|\mathbf{H}\left(\mathbf{F}_{\psi}\right)\right|}_{\text {Iteration Equation Type 2 }}
\end{array}\right.
$$

Then, after comparing the right-hand sides of Eq. (7) and (8a), it is concluded that

$$
\left\{\frac{\partial \mathbf{f}\left(\mathbf{F}_{\psi}\right)}{\partial F_{j}}\right\}_{\max }=\frac{\partial \mathbf{g}\left(\mathbf{F}_{\psi}\right)}{\partial F_{j}}=\left[\begin{array}{c}
\frac{\partial g_{1}\left(\mathbf{F}_{\psi}\right)}{\partial F_{j}} \\
\vdots \\
\frac{\partial g_{i}\left(\mathbf{F}_{\psi}\right)}{\partial F_{j}} \\
\vdots \\
\frac{\partial g_{n}\left(\mathbf{F}_{\psi}\right)}{\partial F_{j}}
\end{array}\right]
$$

Now, the partial derivatives of Eq. (7) can be described by using column vector $\mathbf{g}\left(\mathbf{F}_{\psi}\right)$ instead of column vector $\mathbf{f}\left(\mathbf{F}_{\psi}\right)$. The corresponding central difference formula for the $i$-th row of column vector $\mathbf{g}\left(\mathbf{F}_{\psi}\right)$ can be expressed as follows ...

$$
\left\{\frac{\partial f_{i}\left(\mathbf{F}_{\psi}\right)}{\partial F_{j}}\right\}_{\max }=\frac{\partial g_{i}\left(\mathbf{F}_{\psi}\right)}{\partial F_{j}} \approx \frac{g_{i_{(+)}}-g_{i_{(-)}}}{2 \cdot \Delta F_{j}}
$$

where

$$
\begin{aligned}
g_{i_{(+)}} & =g_{i}\left(F_{\psi_{1}}, \ldots, F_{\psi_{j-1}}, F_{\psi_{j}}+\Delta F_{j}, F_{\psi_{j+1}}, \ldots, F_{\psi_{n}}\right) \\
g_{i_{(-)}} & =g_{i}\left(F_{\psi_{1}}, \ldots, F_{\psi_{j-1}}, F_{\psi_{j}}-\Delta F_{j}, F_{\psi_{j+1}}, \ldots, F_{\psi_{n}}\right) \\
\Delta F_{j} & \equiv \text { step size of load component } F_{j}
\end{aligned}
$$

In general, it is known that the accuracy of the central difference formula is influenced by the roundoff error in the step size $\Delta F_{j}$. Therefore, the step size $\Delta F_{j}$ has to be carefully selected such that $F_{\psi_{j}}$ and $F_{\psi_{j}} \pm \Delta F_{j}$ differ by an exactly representable number. An optimal choice for the step size $\Delta F_{j}$ exists that is a function of a characteristic scale $\lambda$ and the relative machine precision $\varepsilon$. The optimal choice of $\Delta F_{j}$ for the central difference formula is given by the expression (taken from Ref. [7], p. 182, Eq. (5.7.8))

$$
\Delta F_{j} \approx \lambda \cdot \varepsilon^{1 / 3}
$$

where $\lambda$ is the characteristic scale and $\varepsilon$ is the relative machine precision at runtime. Estimates of the characteristic scale $\lambda$ and the relative machine precision $\varepsilon$ are needed if Eq. (10a) is to be applied. The 
scalar value of the perturbed variable itself, i.e., $F_{\psi_{j}}$, is a good choice for $\lambda$ in our context because it is a positive number that is related to the range of the $j$-th load component. Therefore, we can write:

$$
\lambda=F_{\psi_{j}}
$$

In addition, the Forsythe, Malcolm, and Moler algorithm may be used to determine the relative machine precision $\varepsilon$ at runtime (see Ref. [8], pp. 13-14). This simple algorithm computes an approximate value for the smallest quantity such that $(1+\varepsilon)$ is greater than 1 in floating point arithmetic. An implementation of the algorithm as a function call could look as follows assuming that the IDL programming language is used:

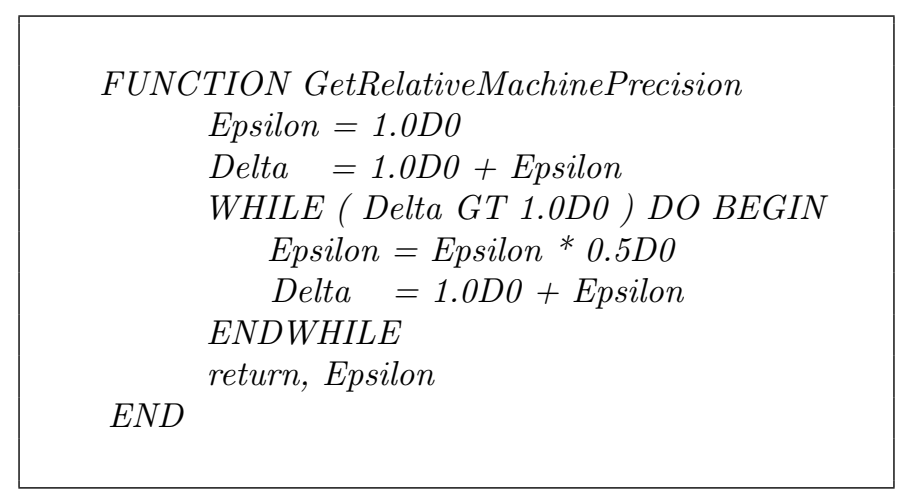

A formula for the lower bound of the number of iterations is derived and discussed in great detail in the next section of the paper.

\section{Lower Bound of Number Of Iterations}

It is possible to compute a theoretical estimate of the lower bound of the number of iterations needed to satisfy a chosen load iteration tolerance. The required relationship can be derived by using a related inequality as a starting point. This inequality describes a connection between the chosen load iteration equation, the desired load iteration tolerance, the upper bound of the Lipschitz constant, and the iteration step (the validity of the inequality is rigorously proven in Ref. [6], p. 99-100). In our context, the inequality can be written as follows (see also Ref. [6], p. 99, Eq. $(5-9)$ ) ...

$$
\left\|\mathbf{F}_{\xi}-\mathbf{F}_{\infty}\right\| \leq \frac{\left(L_{\max }\right)^{\xi}}{1-L_{\max }} \cdot\left\|\mathbf{F}_{\eta}-\mathbf{F}_{\mu}\right\|
$$

where the exponent $\xi$ is the load iteration step, $L_{\max }$ is the upper bound of the Lipschitz constant, $\left\|\mathbf{F}_{\xi}-\mathbf{F}_{\infty}\right\|$ is the Euclidean norm of a load vector difference that is associated with the lower bound of the load change, and $\left\|\mathbf{F}_{\eta}-\mathbf{F}_{\mu}\right\|$ is the Euclidean norm of a load vector difference that is associated with the upper bound of the load change. For completeness, an exact definition of the Euclidean norm of a vector needs to be provided. The Euclidean norm $\|\mathbf{X}\|$ of a $m$-component vector $\mathbf{X}$ is defined as follows:

$$
\mathbf{X}=\left[\begin{array}{c}
x_{1} \\
\vdots \\
x_{k} \\
\vdots \\
x_{m}
\end{array}\right] \Longrightarrow\|\mathbf{X}\|=\sqrt{x_{1}^{2}+x_{2}^{2}+\ldots+x_{k}^{2}+\ldots+x_{m}^{2}}
$$

Now, after solving Eq. (11a) for the exponent, i.e., for the load iteration step $\xi$, we get the following inequality that describes the lower bound of the number of required load iterations: 
LOWER BOUND OF THE NUMBER OF REQUIRED LOAD ITERATIONS

$$
\xi \geq \frac{\ln \left\{\left(1-L_{\max }\right) \cdot \frac{\left\|\mathbf{F}_{\xi}-\mathbf{F}_{\infty}\right\|}{\left\|\mathbf{F}_{\eta}-\mathbf{F}_{\mu}\right\|}\right\}}{\ln \left\{L_{\max }\right\}}
$$

The upper bound $L_{\max }$ of the Lipschitz constant was already defined in Eq. (4). It remains to specify the lower and upper bounds of the load change that is used in Eq. (12) above. The lower bound can be related to the desired load iteration tolerance $\Theta$ (typically $0.0001 \%$ of the upper bound of the loads) and the Euclidean norm of the upper bound $\mathbf{F}_{\psi}$ of the load vector that is defined in Eq. (5). Then, we get:

$$
\begin{gathered}
\left\|\mathbf{F}_{\xi}-\mathbf{F}_{\infty}\right\| \approx \frac{\Theta}{100} \cdot\left\|\mathbf{F}_{\psi}\right\| \\
\text { where } \\
\Theta \equiv \text { tolerance }=0.0001 \%
\end{gathered}
$$

Similarly, the upper bound can be related to the Euclidean norm of the load change that is obtained by using the upper bound $\mathbf{F}_{\psi}$ of the load vector as input on the right-hand side of Eq. (2) (the constants $\mathbf{C}_{\mathbf{1}}^{-1} \Delta \mathbf{R}$ and $\mathbf{B}_{\mathbf{1}}^{-1} \Delta \mathbf{R}$ of the iteration equations are ignored because a constant disappears if the difference of two load vectors is computed). Consequently, we get the following estimate of the upper bound:

$$
\left\|\mathbf{F}_{\eta}-\mathbf{F}_{\mu}\right\| \leq\left\{\begin{array}{l}
\underbrace{\left\|\mathbf{C}_{\mathbf{1}}^{-1} \mathbf{C}_{\mathbf{2}} \cdot \mathbf{H}\left(\mathbf{F}_{\psi}\right)\right\|}_{\text {Iteration Equation Type 1 }} \\
\underbrace{\left\|\mathbf{B}_{\mathbf{1}}^{-1} \mathbf{B}_{\mathbf{2}} \cdot \mathbf{F}_{\psi}+\mathbf{B}_{\mathbf{1}}^{-1} \mathbf{C}_{\mathbf{2}} \cdot \mathbf{H}\left(\mathbf{F}_{\psi}\right)\right\|}_{\text {Iteration Equation Type } 2}
\end{array}\right.
$$

Data from the machine calibration of a six-component force balance is used in the next section to illustrate the use of the iteration convergence test.

\section{Discussion of Example}

Data from a machine calibration of NASA's MC60E balance is used to illustrate the application of the iteration convergence test. The MC60E balance is a six-component force balance that was manufactured by Calspan Force Measurement Systems (San Diego, California). It has a diameter of 2.0 inches and measures five forces and one moment (N1, N2, S1, S2, AF, RM). Table 2 below lists load capacities of the balance:

Table 2: Load capacities of the 2.0 inch MC60E force balance.

\begin{tabular}{|c|c|c|c|c|c|c|}
\hline & N1, lbs & N2, lbs & S1, lbs & S2, lbs & RM, in-lbs & AF, lbs \\
\hline \hline Capacity & 2500 & 2500 & 1250 & 1250 & 5000 & 700 \\
\hline
\end{tabular}


The chosen machine calibration data set was obtained in 2016 in FMS' Automatic Balance Calibration System (ABCS). The final data set consisted of 2091 points that were distributed across 16 load series. Both single and multi-component loadings were applied during the calibration that were defined using a recently developed machine load schedule design (see Ref. [9] for more details). In theory, this load schedule design can support up to 96 math terms and the intercept term in a regression model of a gage output if massive near-linear dependencies between the regression model terms are temporarily ignored.

The modified and extended load iteration convergence test was implemented in NASA's BALFIT software package that is used to analyze strain-gage balance data (see Ref. [10] for more details about the BALFIT software). Therefore, it was possible to process and compare six different analysis choices (cases) of the chosen machine calibration data set in order to illustrate a variety of convergence test results.

First, the calibration data was analyzed using iteration equation Type 1 in combination with a standard 28 -term math model that does not use any absolute value or third order terms ( 28 terms $\equiv 27$ terms plus the intercept). This math model is the baseline model for the balance as none of its gages shows "bi-directional" behavior that would support the use of absolute value terms. Figure 1a shows results of this case for the three possible choices of the upper bound of the load vector. In all cases, the upper bound of the Lipschitz constant is well below the threshold of one. In addition, the convergence is rapid as the estimated lower bound of the number of iterations is below 10 .

Figure $1 \mathrm{~b}$ shows test results if iteration equation Type 1 is used in combination with a 97 -term math model. It can be seen that the upper bounds of the Lipschitz constant are substantially larger than values reported in Fig. 1a for the first analysis situation. This observation seems to indicate that the iteration is closer to being divergent. This conclusion is associated with the fact that the 97 -term math model has very large near-linear dependencies (i.e., a variance inflation factor maximum of 290) that put the uniqueness of the load predictions into question.

Figure 1c shows convergence test results if iteration equation Type 2 instead of Type 1 is used in combination with the 28-term math model. It is observed that the convergence is not as rapid as it was when Type 1 was used (compare test results listed in Fig. 1a with results listed in Fig. 1c). This result is supported by the fact that the actual calculation of the fitted 2091 calibration loads required 2 iterations for Type 1 and 4 iterations for Type 2 in order to meet the tolerance of $0.0001 \%$ of capacity.

Figure 1d shows iteration convergence test results if iteration equation Type 1 is used in combination with a regression model term reduction algorithm that only retains the statistically most significant terms. The upper bound of the Lipschitz constant is smaller when compared with results that are reported in Fig. 1a. This result is expected because a reduction of the number of regression model terms will reduce the upper bound of the Lipschitz constant.

It was decided to also analyze the MC60E calibration data after the balance loads were converted from force balance to direct-read format. Figure $2 \mathrm{a}$ shows corresponding convergence test results if iteration equation Type 1 is used again with a 28-term math model. In all cases, i.e., for all three load vector choices, the upper bound of the Lipschitz constant is well below the threshold of one. However, the convergence is not as rapid as it was when the data was analyzed in force balance format because the estimated lower bound of the number of iterations is near or greater than 10 .

Finally, the converted MC60E calibration data set was analyzed by using iteration equation Type 2 instead of iteration equation Type 1. Test results for this case are listed in Fig. 2b. The iteration convergence test failed for all three upper bounds of the load vector. This result is expected as iteration equation Type 2 will, by design, only work if strain-gage balance data is analyzed in its "design format" (see Ref. [4] for a detailed discussion of this limitation of iteration equation Type 2).

\section{Summary}

A convergence test for iterative wind tunnel strain-gage balance load predictions was discussed. First, the convergence test itself and recent modifications of the test were described. These modifications make it easier to implement the test in a balance data analysis software package. In particular, it is suggested to use numerical instead of explicit differentiation in order to compute some of the convergence test's inputs. Finally, data from the machine calibration of a six-component force balance was analyzed in order to illustrate the application of the iteration convergence test to "real-world" balance data.

American Institute of Aeronautics and Astronautics 


\section{Acknowledgements}

The author wants to thank Tom Volden of Jacobs Technology and Bob Gisler of NASA Ames Research Center for their critical and constructive review of the final manuscript. The work reported in this paper was partially supported by the Wind Tunnel Division at NASA Ames Research Center under contract NNA16BD26C.

\section{References}

[1] AIAA/GTTC Internal Balance Technology Working Group, Recommended Practice - Calibration and Use of Internal Strain-Gage Balances with Application to Wind Tunnel Testing, AIAA R-091-2003, American Institute of Aeronautics and Astronautics, Reston, Virginia, 2003.

[2] Ulbrich, N., and Volden, T., "Strain-Gage Balance Calibration Analysis Using Automatically Selected Math Models," AIAA 2005-4084, paper presented at the 41st AIAA/ASME/SAE/ASEE Joint Propulsion Conference, Tucson, Arizona, July 2005.

[3] Ulbrich, N., and Volden, T., "Application Of A New Calibration Analysis Process to the MK-III-C Balance," AIAA 2006-0517, paper presented at the 44th AIAA Aerospace Sciences Meeting, Reno, Nevada, January 2006.

[4] Ulbrich, N., "Influence of Primary Gage Sensitivities on the Convergence of Balance Load Iterations," AIAA 2012-0322, paper presented at the 50th AIAA Aerospace Sciences Meeting, Nashville, Tennessee, January 2012.

[5] Smith, D. L., An Efficient Algorithm Using Matrix Methods to Solve Wind-Tunnel Force-Balance Equations, NASA Technical Note TN D-6860, Langley Research Center, Hampton, Virginia, August 1972.

[6] Henrici, P., Elements of Numerical Analysis, 1st ed., John Wiley \& Sons, Inc., New York, London, Sydney, 1964, pp. 97-105.

[7] Press, W. H., Teukolsky, S. A., Vetterling, W. T., Flannery, B. P., "Numerical Recipes in FORTRAN 77," Cambridge University Press, reprint of second edition with corrections, 1996, pp. 180-182.

[8] Forsythe, G. E., Malcolm, M. A., Moler, C. B., "Computer Methods for Mathematical Computations," Prentice-Hall, Inc., Englewood Cliffs, New Jersey, 1977, pp. 13-14.

[9] Ulbrich, N., Gisler, R., and Kew, R., "Assessment of New Load Schedules for the Machine Calibration of a Force Balance," AIAA 2015-2023, paper presented at the 53rd AIAA Aerospace Sciences Meeting, Kissimmee, Florida, January 2015.

[10] Ulbrich, N., and Volden, T., BALFIT - Software Tool for the Regression Analysis of Multivariate Data, User Guide (revised and corrected 4th edition), Jacobs Technology Inc., prepared for NASA Ames Research Center under contract NNA09DB39C, February 2016. 


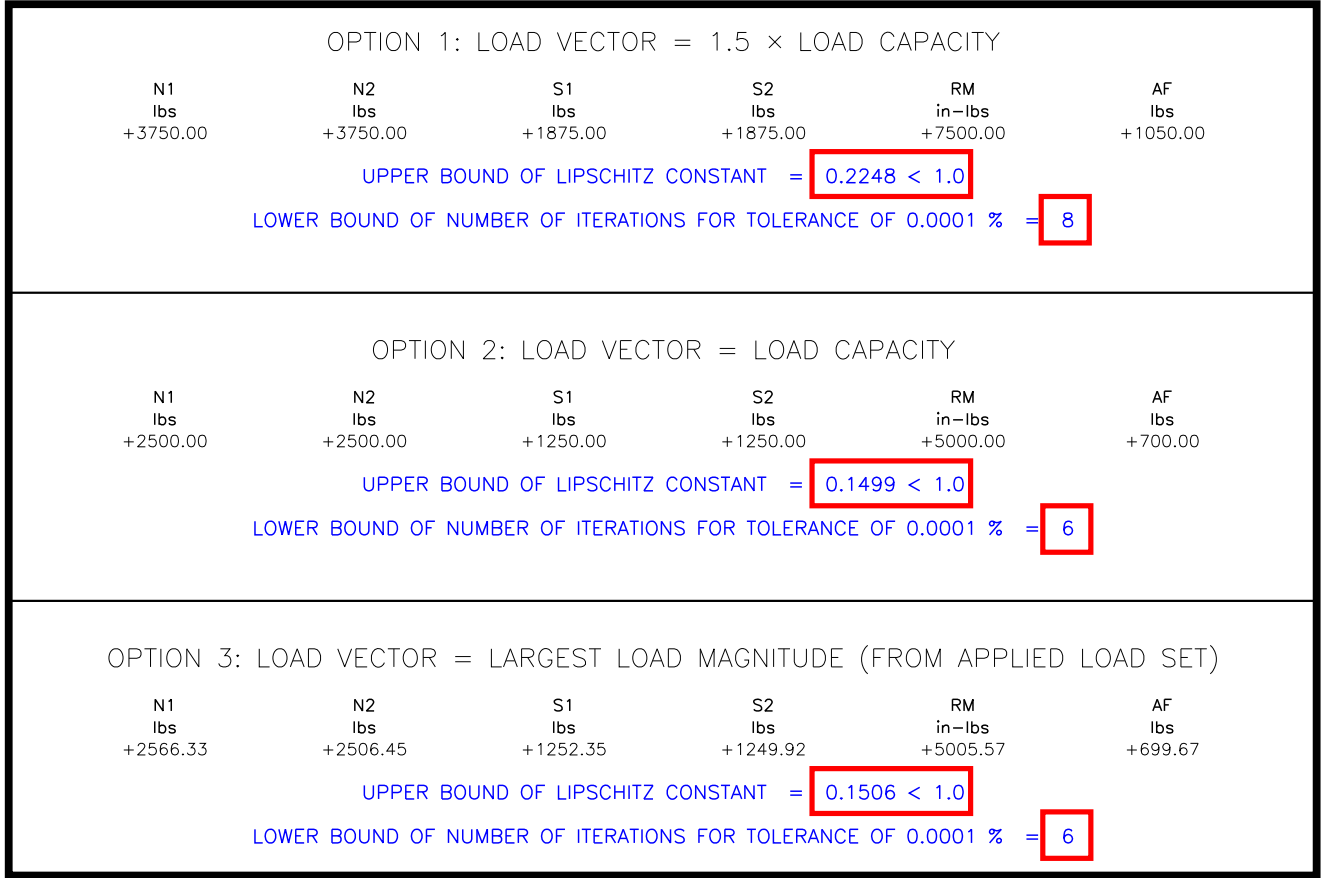

Fig. 1a Load iteration convergence assessment for the NASA's MC60E six-component force balance (Case $1 \Longrightarrow$ Iteration Equation Type 1, 28-term math model $\equiv 27$ terms plus intercept).

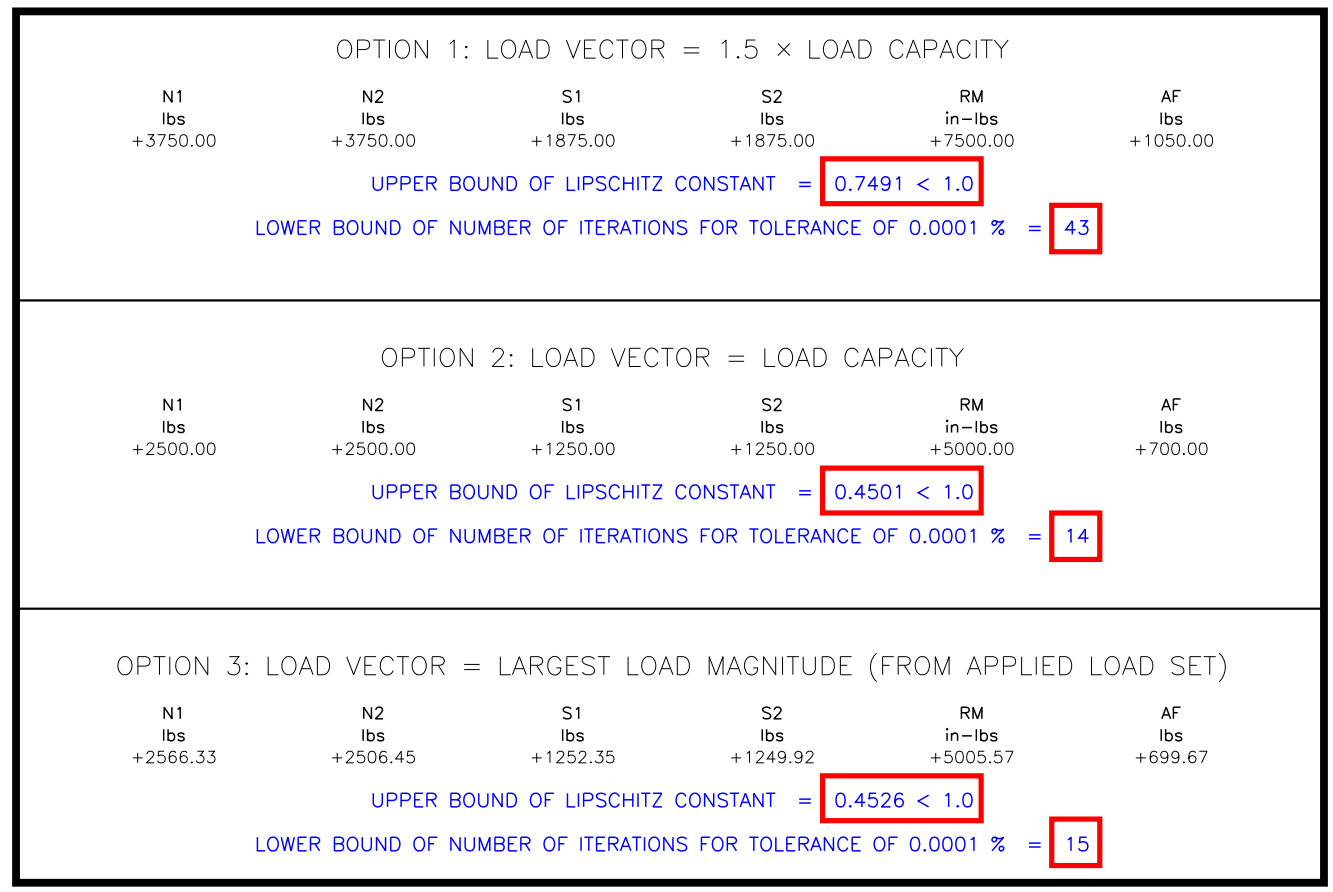

Fig. 1b Load iteration convergence assessment for the NASA's MC60E six-component force balance (Case $2 \Longrightarrow$ Iteration Equation Type 1, 97-term math model $\equiv 96$ terms plus intercept). 


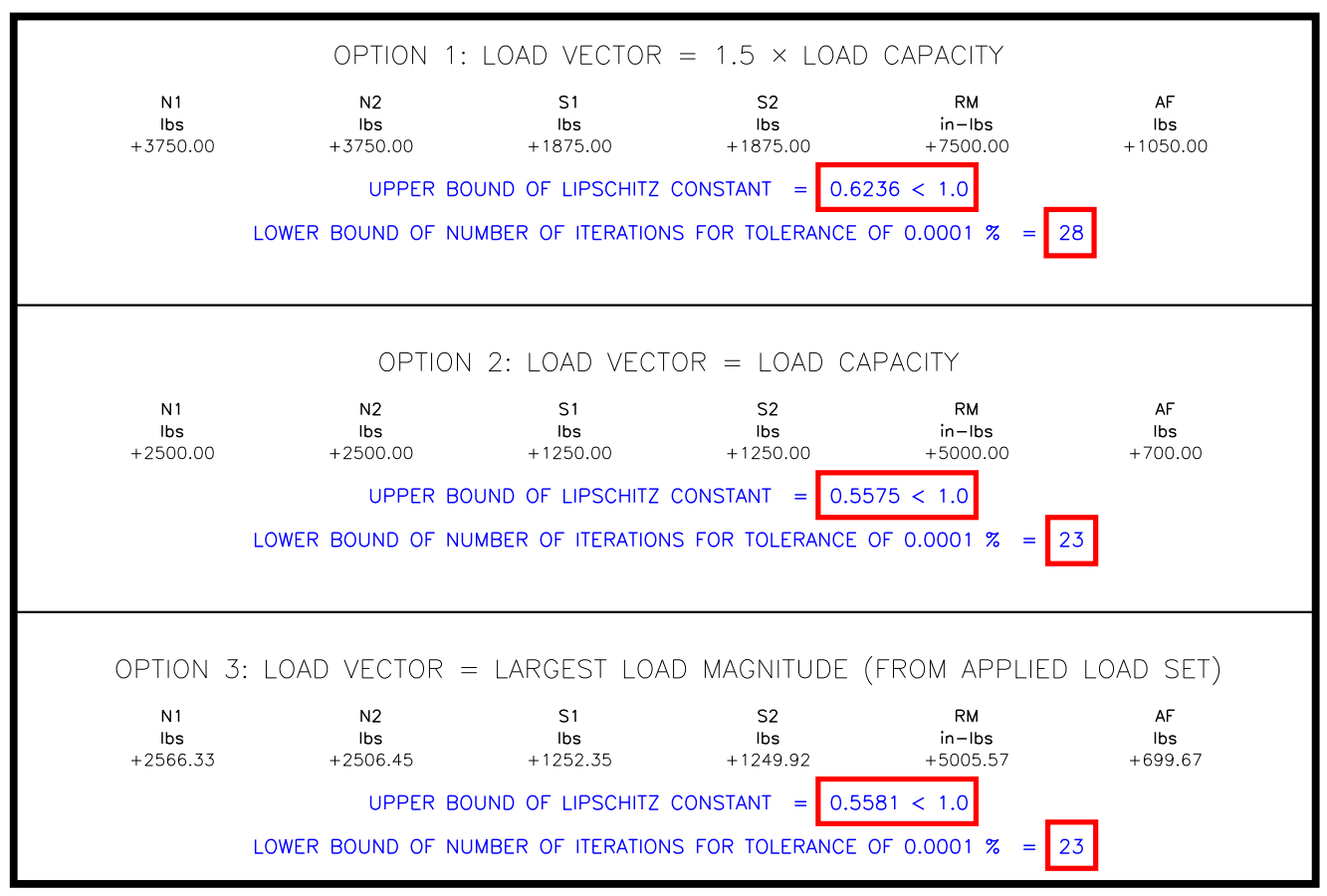

Fig. 1c Load iteration convergence assessment for the NASA's MC60E six-component force balance (Case $3 \Longrightarrow$ Iteration Equation Type 2, 28-term math model $\equiv 27$ terms plus intercept).

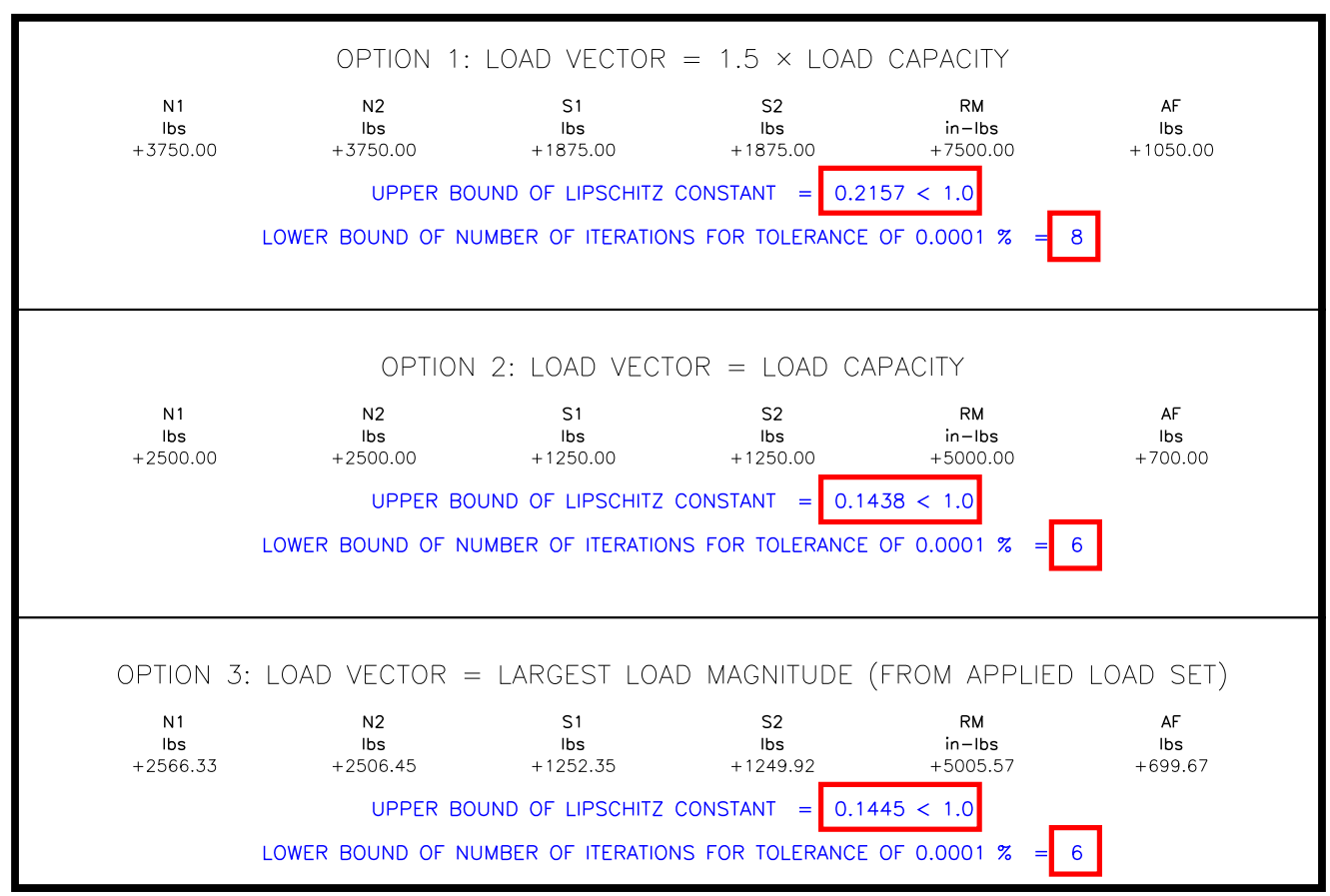

Fig. 1d Load iteration convergence assessment for the NASA's MC60E six-component force balance (Case $4 \Longrightarrow$ Iteration Equation Type 1, term reduction used, between 16 and 23 terms per gage). 


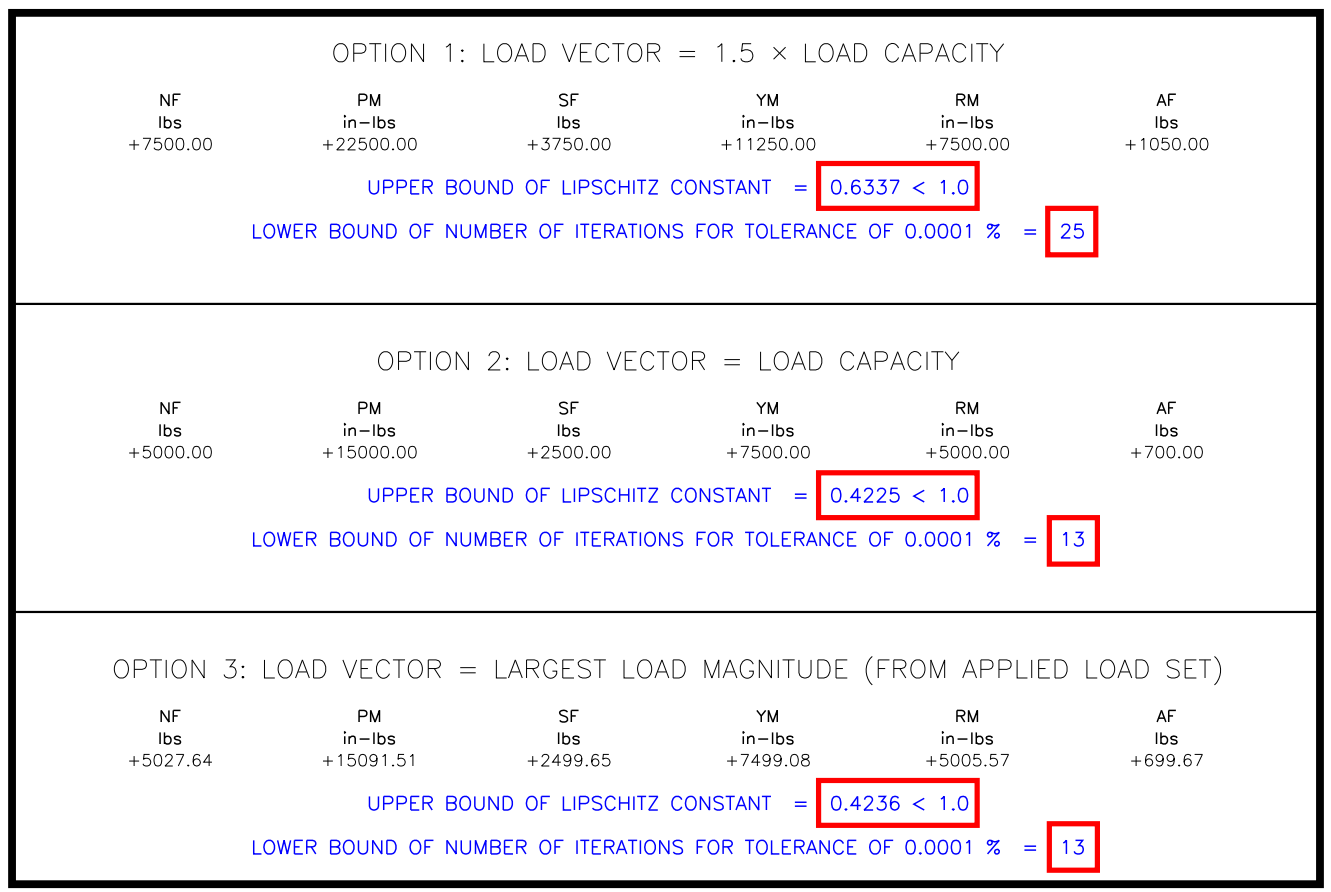

Fig. 2a Load iteration convergence assessment for the NASA's MC60E six-component force balance (Case $5 \Longrightarrow$ Iteration Equation Type 1, loads in direct-read format, i.e, NF, PM, SF, ...).

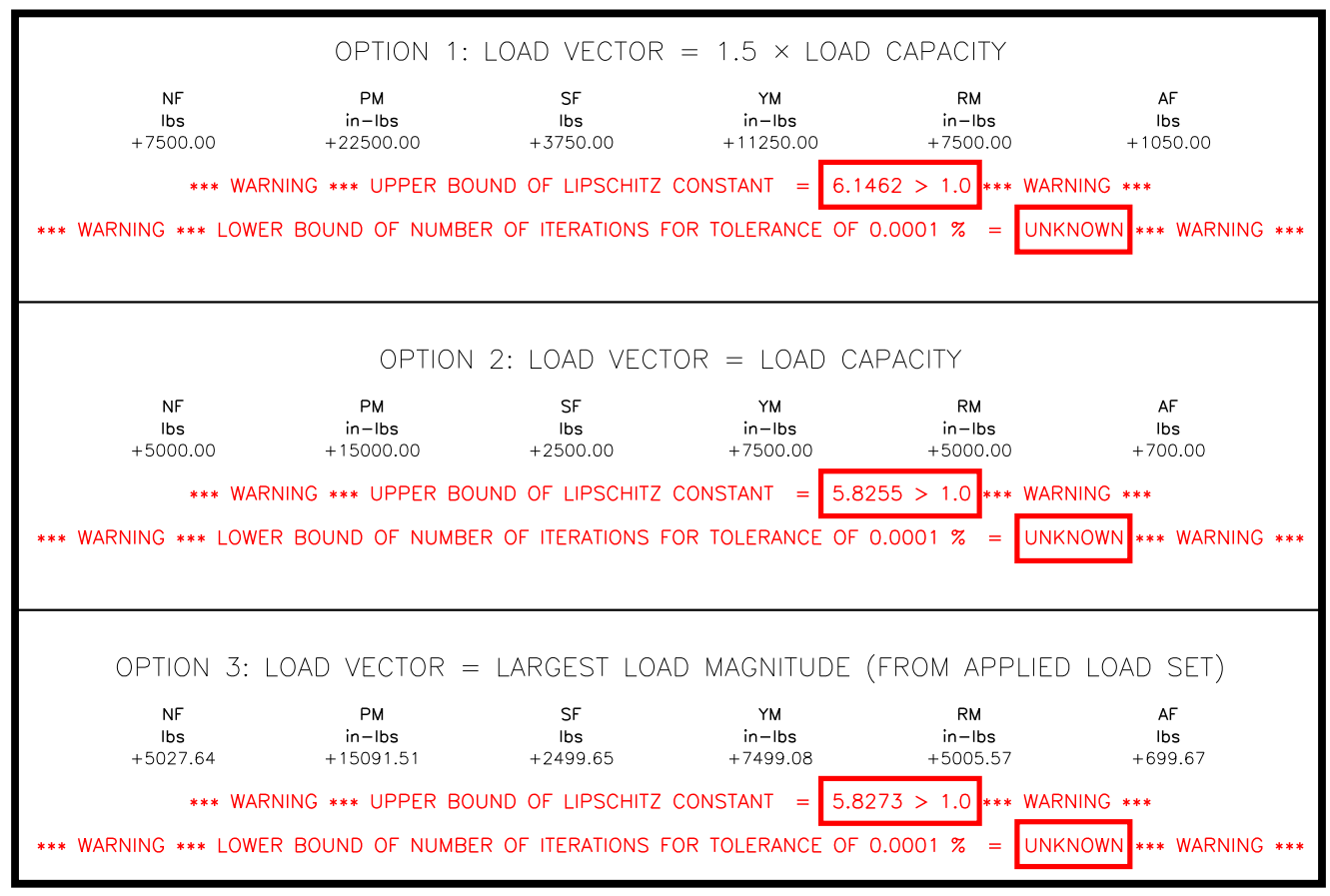

Fig. 2b Load iteration convergence assessment for the NASA's MC60E six-component force balance (Case $6 \Longrightarrow$ Iteration Equation Type 2, loads in direct-read format, i.e, NF, PM, SF, ...). 